\title{
Resection of an endobronchial hamartoma by cryotherapy
}

\author{
Hak-Ryul Kim ${ }^{1}$, Keum-Ha Choi ${ }^{2}$, Eun-Taik Jeong ${ }^{1}$, and Ki-Eun Hwang ${ }^{1}$
}

Departments of ${ }^{1}$ Internal Medicine and ${ }^{2}$ Pathology, Wonkwang University School of Medicine, Iksan, Korea
Received: October 22, 2015 Revised : February 4, 2016 Accepted: February 12, 2016

\section{Correspondence to} Ki-Eun Hwang, M.D.

Tel: $+82-63-859-2584$

Fax: +82-63-855-2025

E-mail: eyesmile@wku.ac.kr
A 72-year-old man was referred to our hospital with an endobronchial mass spotted on chest computed tomography (CT). On examination, he was afebrile, but complained of cough and sputum. Laboratory test results were normal. His chest CT showed an ovoid fat attenuation nodule in the left upper lobe bronchus (Fig. 1A). Flexible bronchoscopy was performed to investigate the endobronchial lesion, and a polypoid tumor was found in the left upper lobe bronchus that was leading to total obstruction (Fig. 1B). Because left upper bronchus obstruction could lead to recurrent postobstructive pneumonia, cryotherapy was performed. The tip of the cryoprobe was placed in direct contact with the tumor and was pulled and withdrawn with the frozen tissue
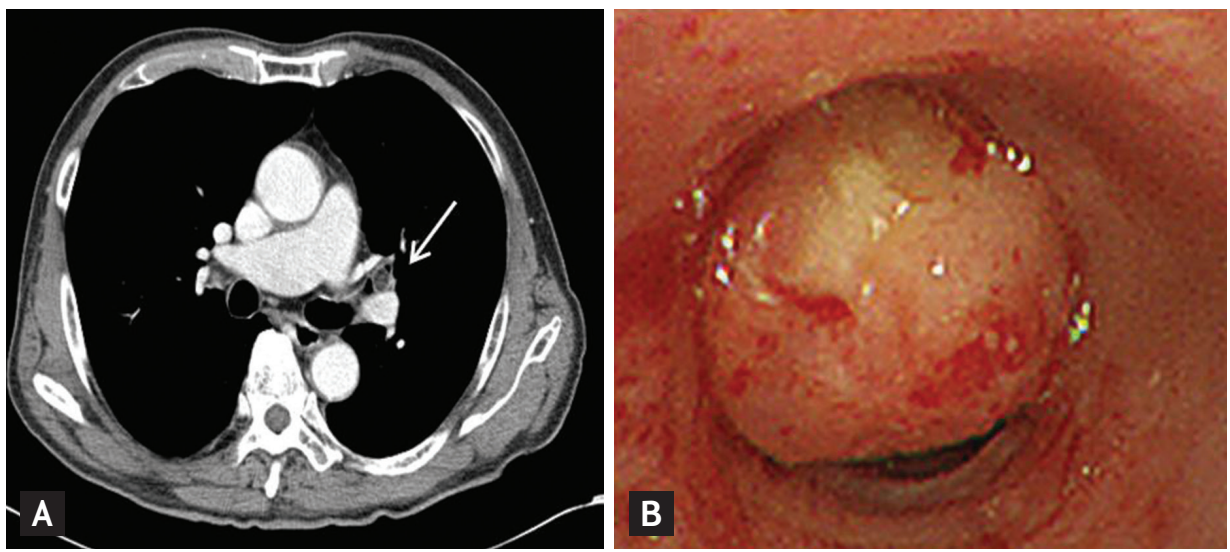

Figure 1. (A) A chest computed tomography (CT) shows a 1.1-cm ovoid fat attenuated nodule in the left upper lobe bronchus (arrow). (B) Bronchoscopic examination shows an intrabronchial tumor in the left upper lobe bronchus. using a bronchoscope (Fig. 2A-2C). The mass was successfully removed after repeated procedures. Histopathological examination showed mature cartilage and adipose tissue in the deeper layer, and the patient was diagnosed with an endobronchial hamartoma (Fig. 2D). At 1-month follow-up, a bronchoscopic examination showed no remnants of the mass and no tumor recurrence.

Hamartomas can occur in any region of the lung, but are rarely identified as endobronchial. A majority of patients with parenchymal hamartoma are asymptomatic; the possibility of endobronchial hamartomas as a cause of postobstructive pneumonia should be considered. In a Korean study that reviewed 24 cases of endobronchial hamartoma, 10 (42\%) showed 


\section{$\mathrm{KJIM} \mathrm{M}^{\mathrm{L}}$}
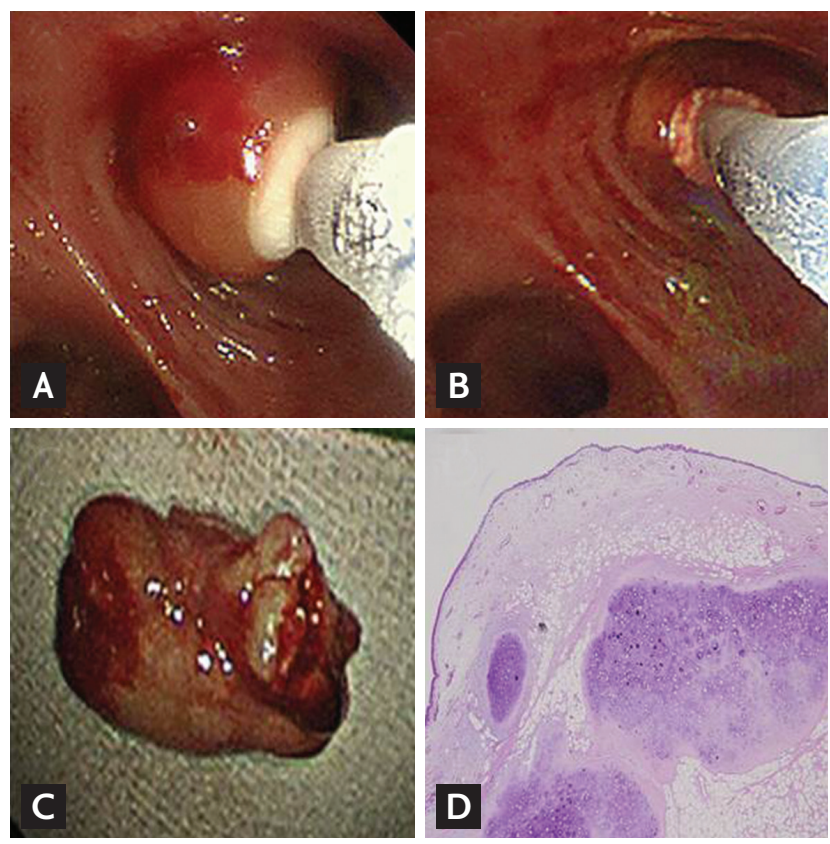

Figure 2. (A-C) Tumor resection using cryotherapy. (D) Histopathology of the tumor shows mature cartilage and adipose tissue (H\&E, ×40).

obstructive pneumonia. Thus, treatment should also be administered to asymptomatic cases. Because ham- artomas rarely recur or become malignant, minimally invasive procedures should be chosen. Bronchoscopy is a modality currently used for diagnosis and treatment. Various bronchoscopic techniques, such as those using YAG laser or argon, electrocautery, cryotherapy, argon, and plasma coagulation are used for benign endobronchial tumors. We herein reported a successfully-treated endobronchial hamartoma using cryotherapy.

In conclusion, endobronchial hamartomas are benign tumors of the tracheobronchial tree that potentially can cause morbidity due to bronchial obstruction and recurrent pneumonia. Endoscopic treatment with a flexible bronchoscope and cryotherapy provide excellent outcomes for these patients.

\section{Conflict of interest}

No potential conflict of interest relevant to this article was reported.

\section{Acknowledgments}

This study was supported by a grant from Wonkwang University in 2014. 